\title{
CHRONIQUES
}

\section{LECTURE ET PHOTOGRAPHIE DES OTOLITHES D'ANGUILLES}

Ayant été affecté à la Région Piscicole de BAR-le-DUC en 1967: j'ai été chargé de capturer à l'élect.icité les anguilles qui pullulaient dans la SEMOIGNE, petit affluent de la rive droite de la MARNE, depuis que dans la pisciculture domaniale de NEUVILLE, située sur cette rive, des civelles avaient èté transitées en grande quantité chaque année de 1953 à 1956 inclus.

La petite taille d'une assez grande proportion des anguilles que j'ai ainsi capturées à partir de 1968, semblait confirmer l'hypothèse qu'avait faite Monsieur I'Ingénieur BACHELIER, lorsqu'il avait eu l'occasion de constater que les petites anguilles de remontee ne cherchaient plus à remonter au pied d'un grand barrage qui s'était opposé totalement à la remontée des anguilles depuis plusieurs décennies: les civelles devenues petites anguilles ne cherchent à remonter que là où sont remontés leurs parents, et de ve fait, les petites anguilles de la SEMOIGNE en 1968, pouvaient être des descendantes des civelles échappées de la Pisciculture Domaniale de NEUVILLE sur la SEMOIGNE de 1953 à 1956.

Cependant leur petitesse po:svait être dùe à une très faible croissance fort explicable dans la SEMOIGNE ou la température des eaux dépasse rarement 110 et par leur nombre excessif; aussi ai-je été chargé par Monsieur I'Ingenieur BACHELIEP de m'instruire sur la détermination des âges des anguilles par la lecture de leurs otolithes.

Une visite aux Services de Monsieur le Professeur FONTAINE, Directeur du Muséum me permit de m'instruire sur l'extraction des Otolithes des anguilles si petites soient-elles et de constater qu'il était déjà possible de distinguer les cernes de croissance dans les otolithes sans préparation. Disposant d'anguilles ide $20 / 25 \mathrm{~cm}$, toutes leurs otolithes semblaient comporter de 8 à 11 cernes, ce qui laissait supposer qu'il ne s'agissait que de civelles de 1956, dont certains cernes d'otolithes passaient inaperçus. 
Ayant pu poursuivre cette étude à l'Institut de Biologie Maritme de WIMEREUX et après de nombreux tâtonnements, j'ai mis au point la méthode suivante pour préparer les otolithes en vue du dénombrement de leurs cernes.

Après extraction de l'otolithe et le plaçant sur mon index. je le polis successivement suivant ses deux grandes faces au moyen d'une pierre INDIAOIL-STONE à grain fin jusqu'à ce que son épaisseur soit d'un demi-millimètre environ, puis j'achève son polissage au moven d'une pierre - ARKANSAS.

Ainsi poli l'otolithe est trempé 5 à 6 secondes dans de l'acide azotique dilué à $40 \%$ puis placé dans une petite cuvette creusée dans un marbre dépoli et contenant quelques gouttes de XYLOL.

L'éclairage est dirigé presque horizontalement pour ne porter oue sur l'otolithe en travers.

La lecture est rendue plus aisee avec un filtre orange ou un filtre polaroïd gris-bleuté.

Pour la photographie il faut utiliser un film de OASA avec le filtre orange, ou un film orthochromatique avec un filtre polaroid gris-bleuté.

Cette méthode appliquée aux petites otolithes (à peine $1 \mathrm{~mm}$ de diamètre) d'anguilles de $15 / 20 \mathrm{~cm}$ récoltées dans le SEMOIGNE en 1969, m'a permis d'estimer les âges de ces individus qui avaient entre 2 et 5 ans d'eau douce et qui, par conséquent, ne pouvaient être que les descendants de civelles échappées dans la SEMOIGNE entre 1953 et 1956.

\author{
J.-M. SIMON \\ Garde-Chef \\ Commissionné de l'Administration
}

\title{
UN RESISTIVIMETRE - CONDUCTIVIMETRE DE TERRAIN PRATIQUE
}

La Société ELECTROPULLMAN (1) diffuse sous le nom de * Résistivimètre-conductivimètre de terrain * un appareil d'un prix modique (actuellement : $518,90 \mathrm{~F}$ TTC.)

Cet appareil expérimenté à la Station de Biarritz, a donné toute satisfaction, tant par ses facilités d'emploi que par sa précision.

A défaut d'un tel appareil, nous avions préconisé de prendre la mesure de la résistivité par la s méthode du tuyau*, dont les résultats étaient relativement peu précis, surtout four les eaux conductrices, et dont la mise en œuvre nécessitait certaines précautions. L'apparition de ce résistivimètreconductivimetre comble donc une grande lacune dans le domaina de l'hydrobiologie.

(1) 5, rue des Bruyères, 92 - Bourg-la-Reine. 